\title{
Outlier Rejection for Diffusion Weighted Imaging
}

\author{
Marc Niethammer ${ }^{1,2,3}$, Sylvain Bouix ${ }^{1,2,3}$, Santiago Aja-Fernández ${ }^{2}$, \\ Carl-Fredrik Westin ${ }^{2}$, and Martha E. Shenton ${ }^{1,3}$ \\ ${ }^{1}$ Psychiatry Neuroimaging Laboratory \\ ${ }^{2}$ Laboratory of Mathematics in Imaging, \\ Brigham and Women's Hospital, Harvard Medical School, Boston MA, USA \\ ${ }^{3}$ Laboratory of Neuroscience, VA Boston Healthcare System, Brockton MA, USA \\ \{marc, sylvain, santi, westin, shenton\}@bwh . harvard. edu
}

\begin{abstract}
This paper introduces an outlier rejection and signal reconstruction method for high angular resolution diffusion weighted imaging. The approach is based on the thresholding of Laplacian measurements over the sphere of the apparent diffusion coefficient profiles defined for a given set of gradient directions. Exemplary results are presented.
\end{abstract}

\section{Motivation and Background}

Diffusion weighted imaging (DWI) has become an increasingly popular imaging technique in neuroscience as it provides insight into the architecture of brain white matter. The imaging technology is ever improving and high angular and spatial resolutions are now possible. However, acquiring many gradient directions and small voxel sizes requires fast imaging techniques such as Echo Planar Imaging (EPI), and these are usually prone to various artifacts and often have low signal to noise ratios. The most common artifacts associated with diffusion EPI are susceptibility artifacts and geometric distortions due to gradient induced eddy currents. Less common, but critical to DWI are strong signal drops introduced by patient motion or scanner table vibrations [1.

Preprocessing of DWI data is thus beneficial when dealing with such data. Typically, diffusion data acquired through EPI receives the following processing steps [2]. First, geometric distortions induced by eddy currents are corrected using affine coregistration to the baseline image. Second, the image may be filtered for noise. Finally, diffusion tensors are estimated at each image location. Image intensity artifacts, like strong signal drops, are sometimes taken into account by a robust tensor estimator based on an outlier rejection mechanism 32. While these methods are powerful, they assume Gaussian diffusion profiles, thus removing some of the benefits of high angular resolution DWI. Various methods exist for noise removal and direct estimation of diffusion profiles for high angular resolution DWI acquisitions (e.g. 4]), however these methods do not explicitly address the rejection of outliers. A robust estimation method in analogy to the work in [3] could be constructed for a richer class of diffusion profiles. We instead present an outlier rejection algorithm that is not tied to any diffusion model,

N. Ayache, S. Ourselin, A. Maeder (Eds.): MICCAI 2007, Part I, LNCS 4791, pp. 161-168, 2007.

(C) Springer-Verlag Berlin Heidelberg 2007 
makes minimal assumptions about the process of magnetic resonance (MR) diffusion, and allows for the use of subsequent processing methods without the need for explicit outlier handling.

\section{Outlier Rejection Approach}

Fig. 1 shows an extreme outlier example, where whole slices for gradient directions exhibit signal intensities that are too low. Since dark values indicate large diffusion, linear least squares tensor estimation may result in incorrect directional information due to dropped slices. Our hypothesis is that at a voxel location, the signal associated with neighboring gradient directions should be relatively smooth on the sphere and that outlier signals exhibit sudden changes which can be detected (Fig. 1(a) and (b)). We quantify this measure of smoothness by the Laplacian, on the sphere, of the apparent diffusion coefficients (ADC). We seek to find voxels exhibiting a large Laplacian (Fig. 1(b)) and replace them by interpolating the values of their neighbors on the sphere. Our processing pipeline decomposes into the following steps: (i) affine registration of the DWI volumes to the baseline volume to account for patient movement and eddy currents. This is done using the FSL packagt; (ii) noise filtering of the aligned volumes (Sec. 2.1); (iii) Laplacian computation of the ADC profile on the sphere for every voxel location (Sec. 2.2); (iv) thresholding of the Laplacian profile to declare outliers

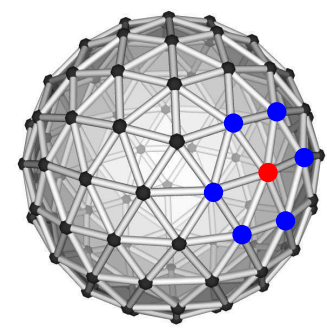

(a) Exemplary gradient directions.

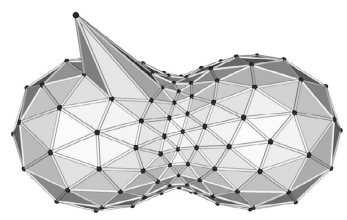

(b) Outlier example.

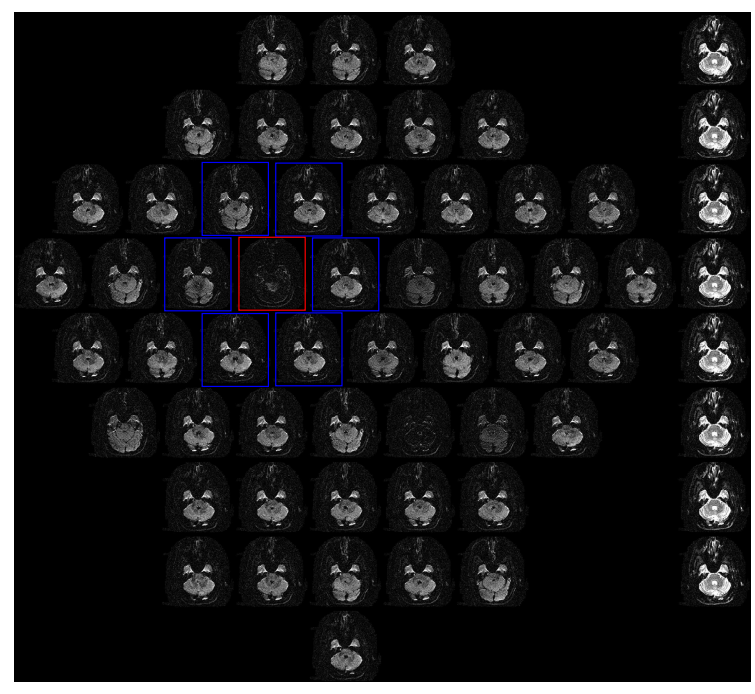

(c) Mapped gradient directions.

Fig. 1. Exemplary outliers, relation to the gradient directions, and an example diffusion profile with an outlier

\footnotetext{
${ }^{1}$ http://www.fmrib.ox.ac.uk/fsl/
} 
(Sec. 2.3); and (v) replacement of image information of potential outliers by sphere-neighborhood-interpolated values (Sec. 2.4).

\subsection{Noise Filtering}

Since the proposed outlier rejection methodology is based on the computation of the Laplacian (a second derivative) noise filtering is an essential pre-processing step to ensure that noise effects will not dominate the Laplacian results as derivative operators amplify noise. Unlike direct filtering of a diffusion profile (e.g. 4]), our noise filtering is not based on a diffusion model, but performed separately on each individual baseline and gradient volumes. A Linear Minimum Mean Square Error Estimator respecting the Rician noise model is used for the filtering [5]. The filtering is performed slice by slice. The estimator is

$$
\widehat{A_{i j}^{2}}=\left\langle M_{i j}^{2}\right\rangle-2 \sigma_{n}^{2}+K_{i j}\left(M_{i j}^{2}-\left\langle M_{i j}\right\rangle^{2}\right),
$$

with $K_{i j}=1-\frac{4 \sigma_{n}^{2}\left(\left\langle M_{i j}^{2}\right\rangle-\sigma_{n}^{2}\right)}{\left\langle M_{i j}^{4}\right\rangle-\left\langle M_{i j}^{2}\right\rangle^{2}}$, where $(\cdot)_{i j}$ denotes position $(i, j), M_{i j}$ is the image

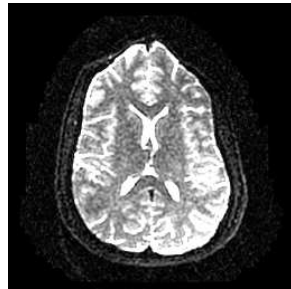

(a) Original baseline.

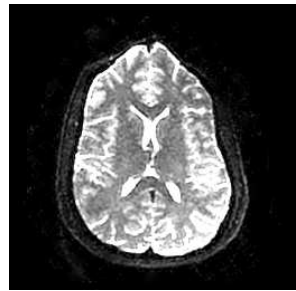

(b) Smoothed baseline.

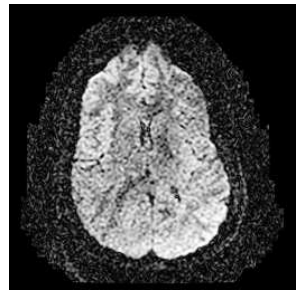

(c) Original DWI.

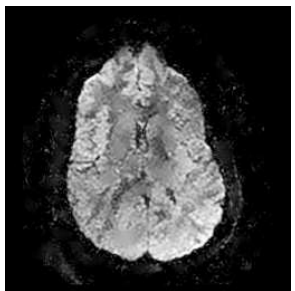

(d) Smoothed DWI.

Fig. 2. Smoothing before Laplacian calculations helps removing the noise without greatly affecting the ability to detect outliers. In accordance with the Rician noise model dark areas are smoothed more than bright areas.

intensity magnitude, $A_{i j}$ the noise-free image intensity magnitude, $\widehat{(\cdot)}$ denotes estimated quantities, $\langle\cdot\rangle$ is the sample estimated value operator, and $\sigma_{n}$ is the noise standard deviation of the Rician noise mode 2

$$
p\left(M \mid A, \sigma_{n}\right)=\frac{M}{\sigma_{n}^{2}} e^{-\frac{M^{2}+A^{2}}{2 \sigma_{n}^{2}}} I_{0}\left(\frac{A M}{\sigma_{n}^{2}}\right) h(M),
$$

where $I_{0}(\cdot)$ is the $0^{\text {th }}$ order modified Bessel function and $h(\cdot)$ is the Heaviside step function. The estimated noise-free image intensity magnitude is given by $\sqrt{\widehat{A_{i j}^{2}}}$. While not strictly fulfilling the noise-model after one filtering step, the filtering methodology may be run recursively [5] (for a number of iteration steps) by

${ }^{2}$ We refer the reader to [5] for a detailed description of how $\sigma_{n}$ is automatically estimated, and how the noise is filtered. 
replacing image magnitudes by their estimated noise-free magnitudes and then applying the filter to these results.

Fig. 2 shows an exemplary smoothing result for an axial baseline slice and an axial slice of a DWI volume. As expected from the Rician noise model assumption, dark areas are smoothed to a greater extent. Noise filtering was done with an estimation and filtering neighborhood of 7 by 7 . Fifteen iterations were executed, running the algorithm to steady state. To demonstrate that filtering in the volume domain suggests beneficial effects on Laplacian computations (see Sec. 2.2 on how to compute the Laplacian) Fig. 3 shows a two-dimensional loghistogram, displaying the log-frequency of Laplacian-ADC pairs. Clearly, noise filtering tightens the distribution and results in less pronounced Laplacian-tails of the distribution.

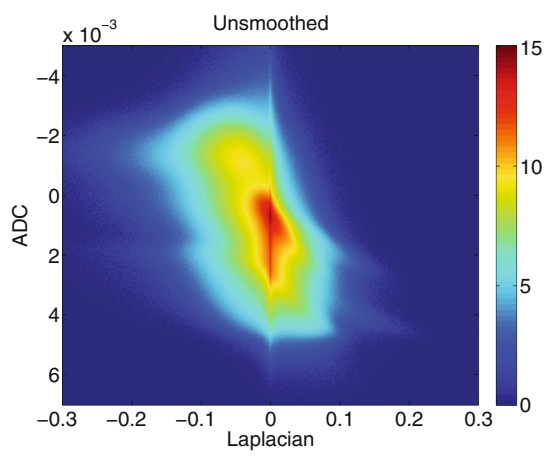

(a) Unsmoothed.

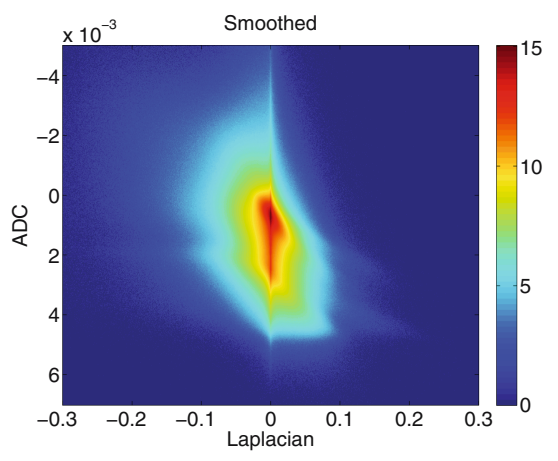

(b) Smoothed.

Fig. 3. Smoothing of DWI volumes reduces the likelihood of obtaining large Laplacian values, suggesting beneficial noise removal properties for the computation of local diffusion profiles

\subsection{Laplace-Beltrami Computations}

Given a set of unit norm gradient direction $\left\{\boldsymbol{g}_{i}\right\}$, we compute the convex hull, $M$, of the set of points given by the gradient directions and its antipodal pairs $\mathcal{P}=$ $\left\{\boldsymbol{g}_{i},-\boldsymbol{g}_{i}\right\}$ to establish neighborhood relations between the gradient directions and to induce a spherical surface triangulation based on the specified gradient directions. To find severe outliers we compute the Laplacian of the ADCs over the sphere. This can be accomplished by means of the Laplace-Beltrami operator $\Delta_{l b}$.

At a given point $\boldsymbol{p}_{i} \in \mathcal{P}$ one can define a discretized version of $\Delta_{l b}$ of the form

$$
\Delta_{l b} \boldsymbol{p}_{i}=\sum_{j \in \mathcal{N}(i)} w_{i j}\left(\boldsymbol{p}_{j}-\boldsymbol{p}_{i}\right)
$$


where $\mathcal{N}(i)$ denotes the one-ring neighborhood set of vertex $\boldsymbol{p}_{i}$, i.e., all vertices that are directly connected to $\boldsymbol{p}_{i}$, and $w_{i j}$ are positive weighting constants given by the discretization scheme, 6]. We use the weighting scheme proposed by Meyer et al. 7]

$$
w_{i j}=\frac{1}{A_{M}\left(\boldsymbol{p}_{i}\right)} \frac{\cot \alpha_{i j}+\cot \beta_{i j}}{2},
$$

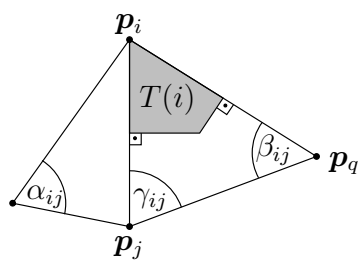

Fig. 4. Illustration of the weight computation for the discretization of the Laplacewhere $A_{M}\left(\boldsymbol{p}_{i}\right)$ denotes the local surface patch of $\boldsymbol{p}_{i}$ and $\alpha_{i j}$ and $\beta_{i j}$ are the angles of the triangles Beltrami operator on a triangulated mesh opposite the edge $(i, j)$, see Fig. 4 for an illustration. $A_{M}\left(\boldsymbol{p}_{i}\right)$ is computed as $A_{M}\left(\boldsymbol{p}_{i}\right)=\sum_{T(i)} \bar{A}(T(i))$, where $T(i)$ denotes the triangles containing vertex $i$ and

$$
\bar{A}(T(i))= \begin{cases}\frac{1}{8}\left(\left\|\boldsymbol{p}_{i}-\boldsymbol{p}_{q}\right\|^{2} \cot \gamma_{i j}+\left\|\boldsymbol{p}_{i}-\boldsymbol{p}_{j}\right\|^{2} \cot \beta_{i j}\right) & \text { if } T(i) \text { is non-obtuse, } \\ \operatorname{area}(T(i)) / 2 & \text { if } T(i) \text { is obtuse at } i \\ \operatorname{area}(T(i)) / 4 & \text { otherwise. }\end{cases}
$$

Using the same discretization weights, we compute the Laplacian of the ADCs on the sphere by applying the discretized $\Delta_{l b}$ to the ADCs associated to the individual gradient directions and their antipodal pairs $\Delta_{l b} a\left(\boldsymbol{p}_{i}\right)=$ $\sum_{j \in \mathcal{N}(i)} w_{i j}\left(a\left(\boldsymbol{p}_{j}\right)-a\left(\boldsymbol{p}_{i}\right)\right)$, where the ADC is given as $a\left(\boldsymbol{p}_{i}\right)=-\frac{1}{b} \log \frac{S\left(\boldsymbol{p}_{i}\right)}{S_{0}}$, with $S_{0}$ the non-diffusion-weighted baseline image intensity, $b$ the b-value and $S\left(\boldsymbol{p}_{i}\right)$ the DWI intensity of the gradient direction associated with the vertex $\boldsymbol{p}_{i}$.

\subsection{Thresholding to Define Outliers}

Once the Laplacian of the ADCs over the sphere has been computed for the whole image volume based on the noise-filtered DWIs, thresholds to declare a measurement as an outlier need to be established. Only large deviations from the norm (decreases as well as increases) should be picked up by the methodology. To define this thresholding region, we create two-dimensional histograms for a number of DWI reference volumes. The histograms measure the frequency of (ADC, Laplacian of ADC) pairs. The histograms for the reference DWI acquisitions are summed up and subsequently smoothed with a Gaussian filter to account for sparse histogram sampling.

Data resulting in negative ADC values are always flagged as outliers and only voxels inside the brain are considered 3 . For all other data, Laplacian thresholds are established for every ADC value. These fixed thresholds are then being applied to newly acquired data. A simple outlier rejection method [8] based on the interquartile range is used; more sophisticated methodologies are conceivable. Specifically, given the observed Laplacian values $(l(A D C))$ for an $\mathrm{ADC}$ value,

\footnotetext{
${ }^{3}$ A coarse brain mask was created by simple intensity thresholding.
} 
the Laplacian outlier thresholds are defined as

$l_{\min }(A D C)=Q_{l}-\frac{3}{2} \mathrm{IQR}, \quad l_{\max }(A D C)=Q_{u}+\frac{3}{2} \mathrm{IQR}, \quad \operatorname{IQR}(A D C)=Q_{u}-Q_{l}$

where $Q_{l}$ and $Q_{u}$ denote the lower and the upper quartiles of $l(A D C)$ and IQR is the interquartile range; see Fig. 5 for an example. All values that are either smaller than $l_{\min }$ or larger than $l_{\max }$ for their Laplacian values are classified as outliers. In this way only extreme values will be considered as outliers.

\subsection{Interpolation}

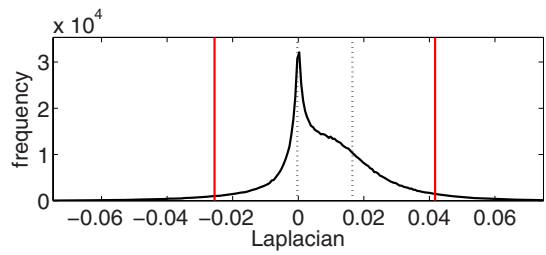

Fig. 5. Example Laplacian histogram for a fixed ADC range. Dotted lines indicate the lower and upper quartiles, solid (red) lines indicate the thresholds $l_{\min }$ and $l_{\max }$ respectively.

Measurements declared as outliers are replaced by smoothly interpolated values based on neighborhood information. Specifically, $\left(a\left(\boldsymbol{p}_{i}\right)\right)_{t}=\Delta_{l b} a\left(\boldsymbol{p}_{i}\right)$, is run to steady state, while keeping all values that are not declared as outliers at their original value throughout the evolution. The ADCs are then mapped back to image intensities.

\section{Results}

We present two experiments, both of which work with data acquired on a GE $3 \mathrm{~T}$ scanner, using an EPI sequence with 51 gradient sensitized directions, 8 baselines and $\mathrm{a} b$ value of $700 \frac{\mathrm{s}}{\mathrm{mm}^{2}}$. The voxel size is $1.7 \mathrm{~mm}^{3}$. Fig. 1 shows an extreme outlier example, where whole slices for gradient directions exhibit signal intensities that are too low. Fig. 6 shows the result of such dropped slices on tensor estimation. Since dark values indicate large diffusion, linear least squares tensor estimation may result in incorrect directional information due to dropped slices. The outlier rejection scheme markedly improves this estimation. Fig. 7 shows results for a less severe, more localized artifact. The resulting reconstruction of the image intensity values is more consistent with its neighborhood and visually sensible.

\section{Conclusion}

We presented a framework for outlier rejection in high angular DWI that does not depend on a specific diffusion model. The method only relies on the weak assumption that measurements in neighboring directions should be similar. We measure this similarity by computing the Laplacian of the ADC on the sphere and find appropriate thresholds to detect and replace outliers. Currently only a simple outlier detection method is used. Future work will look into alternative 


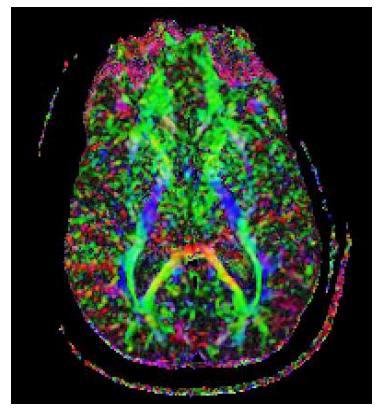

(a) Original slice 40 .

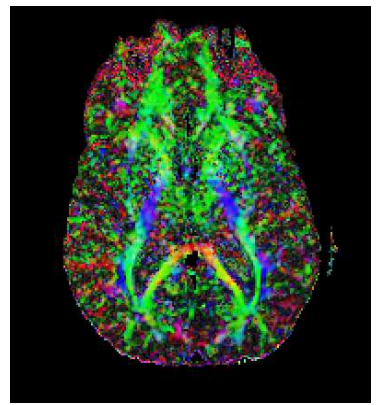

(d) Reconstructed slice 40 .

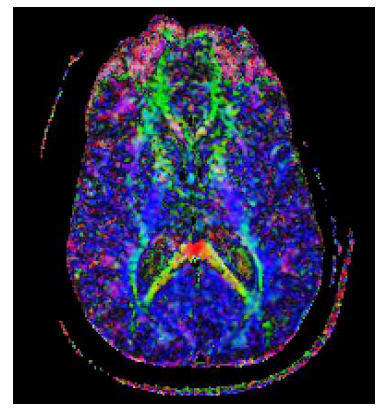

(b) Original slice 41.

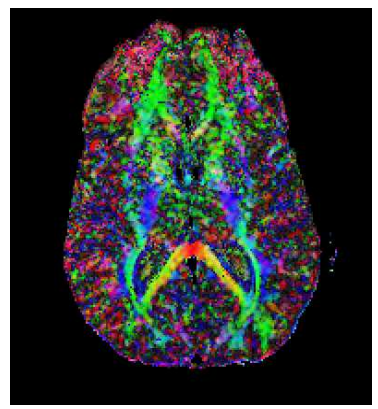

(e) Reconstructed slice 41 .

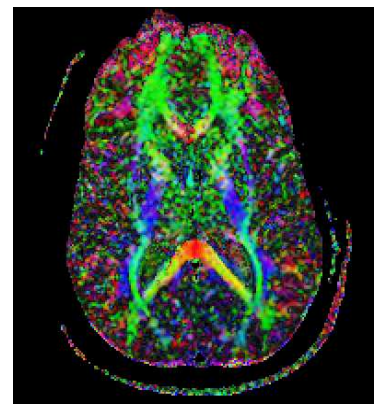

(c) Original slice 42 .

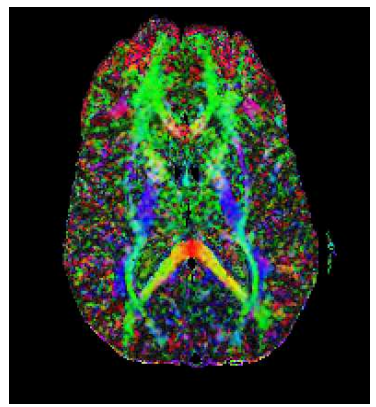

(f) Reconstructed slice 42 .

Fig. 6. Color by orientation from linear least squares tensor estimation with and without outlier rejection. A signal intensity drop can cause major misestimations of diffusion direction (b). After reconstruction, the artifactual blue coloring due to outliers in (b) is replaced by sensible values in (e) through interpolation of ADC on the sphere.

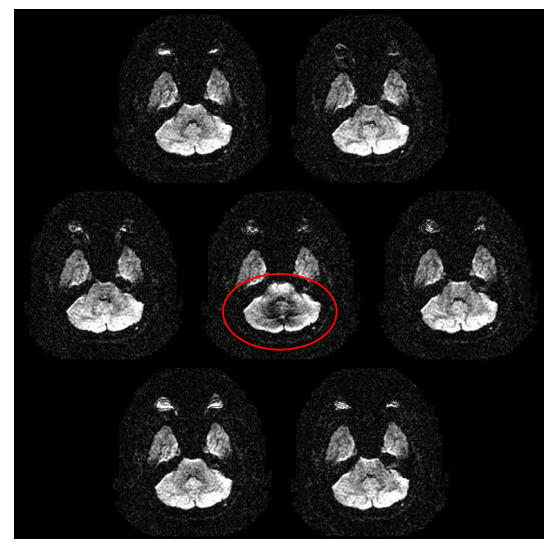

(a) Original.

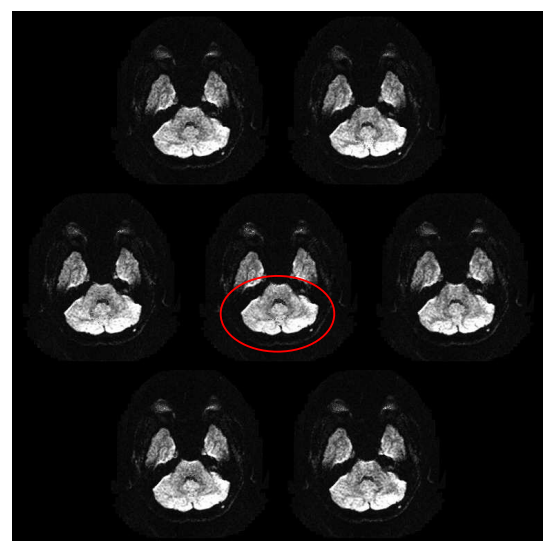

(b) Reconstructed.

Fig. 7. Original and reconstructed DWIs. The outlier rejection and reconstruction methodology succeeds in reconstructing corrupted image information (inside red ellipse) based on the neighboring direction information (the images surrounding the center image). 
outlier detection methodologies. We will also be introducing spatial neighborhood information for the outlier rejection, do an extensive validation of the methodology and will in particular focus on the reconstruction aspect of diffusion profiles that do not follow the tensor model.

\section{Acknowledgements}

This work was supported in part by a Department of Veteran Affairs Merit Award (SB,MS,CFW), a Research Enhancement Award Program (MS), and National Institute of Health grants R01 MH50747 (SB,MS), K05 MH070047 (MS), U54 EB005149 (MN,SB,MS), R01 MH074794 (CFW), and P41 RR13218 $(\mathrm{CFW})$. We thank the anonymous reviewers for their suggestions.

\section{References}

1. Bernstein, M.A., King, K.F., Zhou, X.J.: Handbook of MRI pulse sequences. Elsevier, Amsterdam (2004)

2. Mangin, J.-F., Poupon, C., Clark, C.A., Bihan, D.L., Bloch, I.: Distortion correction and robust tensor estimation for MR diffusion imaging. Medical Image Analysis 6, 191-198 (2002)

3. Chang, L.-C., Jones, D.K., Pierpaoli, C.: RESTORE: Robust estimation of tensors by outlier rejection. Magnetic Resonance in Medicine 53, 1088-1095 (2005)

4. Descoteaux, M., Angelino, E., Fitzgibbons, S., Deriche, R.: Apparent diffusion coefficients from high angular resolution diffusion imaging: Estimation and applications. Magnetic Resonance in Medicine 56, 395-410 (2006)

5. Aja-Fernandez, S., Lopez, C.A., Westin, C.F.: Filtering and noise estimation in magnitude MRI and Rician distributed images. IEEE Transactions on Image Processing (submitted 2007)

6. Xu, G.: Convergent discrete Laplace-Beltrami operators over triangular surfaces. In: Proceedings of the Geometric Modeling and Processing Conference, pp. 195-204 (2004)

7. Meyer, M., Desbrun, M., Schröder, P., Barr, A.H.: Discrete differential-geometry operators for triangulated 2-manifolds. In: Proceedings of VisMath 2002 (2002)

8. Hoaglin, D.C., Iglewicz, B., Tukey, J.W.: Performance of some resistant rules for outlier labeling. Journal of the American Statistical Association 81, 991-999 (1986) 